\title{
Properties of barium strontium titanate at millimeter wave frequencies
}

\begin{abstract}
The trend towards using higher millimetre-wave frequencies for communication systems has created a need for accurate characterization of materials to be used at these frequencies. Barium Strontium Titanate (BST) is a ferroelectric material whose permittivity is known to change as a function of applied electric field and have found varieties of application in electronic and communication field. In this work, new data on the properties of BST characterize using the free space technique at frequencies between $145 \mathrm{GHz}$ and $155 \mathrm{GHz}$ for both thick film and bulk samples are presented. The measurement data provided useful information on effective permittivity and loss tangent for all the BST samples. Data on the material transmission, reflection properties as well as loss will also be presented. The outcome of the work shows through practical measurement, that BST has a high permittivity with moderate losses and the results also shows that BST has suitable properties to be used as RAM for high frequency application.
\end{abstract}

Keyword: BST; Free space measurement; Material characterization; Milimeter-wave 